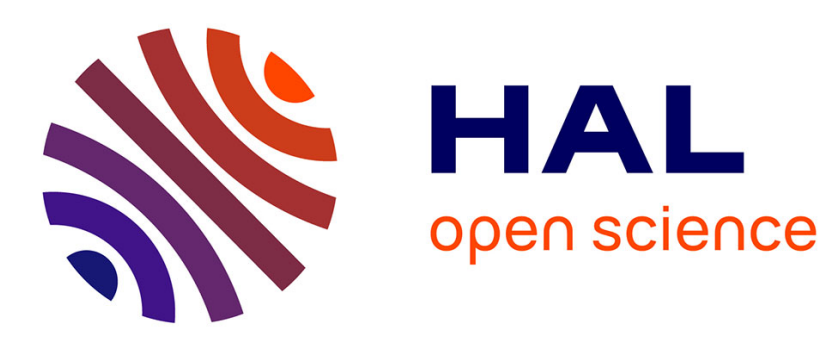

\title{
Limiting the memory footprint when dynamically scheduling DAGs on shared-memory platforms
}

\author{
Loris Marchal, Bertrand Simon, Frédéric Vivien
}

\section{To cite this version:}

Loris Marchal, Bertrand Simon, Frédéric Vivien. Limiting the memory footprint when dynamically scheduling DAGs on shared-memory platforms. Journal of Parallel and Distributed Computing, 2019, 128, pp.30-42. 10.1016/j.jpdc.2019.01.009 . hal-02025521

\section{HAL Id: hal-02025521 \\ https://hal.inria.fr/hal-02025521}

Submitted on 19 Feb 2019

HAL is a multi-disciplinary open access archive for the deposit and dissemination of scientific research documents, whether they are published or not. The documents may come from teaching and research institutions in France or abroad, or from public or private research centers.
L'archive ouverte pluridisciplinaire HAL, est destinée au dépôt et à la diffusion de documents scientifiques de niveau recherche, publiés ou non, émanant des établissements d'enseignement et de recherche français ou étrangers, des laboratoires publics ou privés. 


\title{
Limiting the memory footprint when dynamically scheduling DAGs on shared-memory platforms
}

\author{
Loris Marchal $^{\mathrm{a}}$, Bertrand Simon ${ }^{\mathrm{a}, \mathrm{b}}$, Frédéric Vivien ${ }^{\mathrm{a}}$ \\ ${ }^{a}$ Univ Lyon, EnsL, UCBL, CNRS, Inria, LIP, F-69342, LYON Cedex 07, France \\ ${ }^{b}$ Department of Mathematics and Computer Science, University of Bremen, Bremen, Germany
}

\begin{abstract}
Scientific workflows are frequently modeled as Directed Acyclic Graphs (DAGs) of tasks, which represent computational modules and their dependences in the form of data produced by a task and used by another one. This formulation allows the use of runtime systems which dynamically allocate tasks onto the resources of increasingly complex computing platforms. However, for some workflows, such a dynamic schedule may run out of memory by processing too many tasks simultaneously. This paper focuses on the problem of transforming such a DAG to prevent memory shortage, and concentrates on shared memory platforms. We first propose a simple model of DAGs which is expressive enough to emulate complex memory behaviors. We then exhibit a polynomial-time algorithm that computes the maximum peak memory of a DAG, that is, the maximum memory needed by any parallel schedule. We consider the problem of reducing this maximum peak memory to make it smaller than a given bound. Our solution consists in adding new fictitious edges, while trying to minimize the critical path of the graph. After proving that this problem is NP-complete, we provide an ILP solution as well as several heuristic strategies that are thoroughly compared by simulation on synthetic DAGs modeling actual computational workflows. We show that on most instances we are able to decrease the maximum peak memory at the cost of a small increase in the critical path, thus with little impact on the quality of the final parallel schedule.
\end{abstract}

Keywords: Scheduling, Task graph, Bounded Memory

Email address: loris.marchal@ens-lyon.fr (Loris Marchal) 


\section{Introduction}

Parallel workloads are often described by Directed Acyclic task Graphs, where nodes represent tasks and edges represent dependences between tasks. The interest of this formalism is twofold: it has been widely studied in theoretical scheduling literature [1] and dynamic runtime schedulers (e.g., StarPU [2], XKAAPI [3], StarSs [4], and PaRSEC [5]) are increasingly popular to schedule them on modern computing platforms, as they alleviate the difficulty of using heterogeneous computing platforms. Concerning task graph scheduling, one of the main objectives that have been considered in the literature consists in minimizing the makespan, or total completion time. However, with the increase of the size of the data to be processed, the memory footprint of the application can have a dramatic impact on the algorithm execution time, and thus needs to be optimized [6, 7]. This is best exemplified with an application which, depending on the way it is scheduled, will either fit in the memory, or will require the use of swap mechanisms or out-of-core execution. There are few existing studies that take into account memory footprint when scheduling task graphs, as detailed below in the related work section.

Our focus here concerns the execution of highly-parallel applications on a shared-memory platform. Depending on the scheduling choices, the computation of a given task graph may or may not fit into the available memory. The goal is then to find the most suitable schedule (e.g., one that minimizes the makespan) among the schedules that fit into the available memory. A possible strategy is to design a static schedule before the computation starts, based on the predicted task durations and data sizes involved in the computation. However, there is little chance that such a static strategy would reach high performance: task duration estimates are known to be inaccurate, data transfers on the platform are hard to correctly model, and the resulting small estimation errors are likely to accumulate and to cause large delays. Thus, most practical schedulers such as the runtime systems cited above rely on $d y$ namic scheduling, where task allocations and their execution order are decided at runtime, based on the system state. 
The risk with dynamic scheduling, however, is the simultaneous scheduling of a set of tasks whose total memory requirement exceeds the available memory, a situation that could induce a severe performance degradation. Our aim is both to enable dynamic scheduling of task graphs with memory requirements and to guarantee that the available memory is never exceeded during the execution. We achieve this goal by modifying the input graph in a way that prevents dynamic schedulers from exceeding the memory. Specifically, we add fictitious dependences in the graph: these additional edges will restrict the set of valid schedules and in particular forbid the concurrent execution of too many memory-intensive tasks. This idea is inspired by [8], which applies a similar technique to graphs of smaller-grain tasks. The main difference with the present study is that they focus on homogeneous data sizes: all the data have size 1, which is also a classical assumption in instruction graphs produced by the compilation of programs. On the contrary, our approach is designed for larger-grain tasks appearing in scientific workflows whose sizes are highly irregular.

The rest of the paper is organized as follows. We first briefly review the existing work on memory-aware task graph scheduling (Section 2). We propose a very simple task graph model which both accurately describes complex memory behaviors and is amenable to memory optimization (Section 3). We introduce the notion of the maximum peak memory of a workflow: this is the maximum peak memory of any (sequential or) parallel execution of the workflow. We then show that the maximum peak memory of a workflow is exactly the weight of a special cut in this workflow, called the maximum topological cut. Finally, we propose a polynomial-time algorithm to compute this cut (Section 4). In order to cope with limited memory, we formally state the problem of adding edges to a graph to decrease its maximum peak memory, with the objective of not harming too much the makespan of any parallel execution of the resulting graph. We prove this problem NP-hard and propose both an ILP formulation and several heuristics to solve it on practical cases (Section 5). Finally we evaluate the heuristics through simulations on synthetic task graphs produced by classical random workflow generators (Section 6). The simulations show that the two best heuristics have a limited impact on the makespan in most cases, 
and one of them is able to handle all studied workflows.

Note that a preliminary version of this work was presented at the IPDPS' 18 conference [9].

\section{Related work}

Memory and storage have always been limiting parameters for large computations, as outlined by the pioneering work of Sethi and Ullman [10] on register allocation for task trees, modeled as a pebble game. The problem of determining whether a directed acyclic graph can be pebbled with a given number of pebbles (i.e., executed with a given number of registers) has been shown NP-complete by Sethi [11] if no vertex is pebbled more than once (the general problem allowing recomputation, that is, re-pebbling a vertex which have been pebbled before, has been proven PSPACE complete [12]).

This model was later translated to the problem of scheduling a task graph under memory or storage constraints for scientific workflows whose tasks require large I/O data. Such workflows arise in many scientific fields, such as image processing, genomics, and geophysical simulations. In several cases, the underlying task graph is a tree, with all dependences oriented towards the root, which notably simplifies the problem: this is the case for sparse direct solvers [13] but also in quantum chemistry computations [14]. For such trees, memory-aware parallel schedulers have been proposed [15] and the effect of processor mapping on memory consumption have recently been studied [7].

The problem of general task graphs handling large data has been identified by Ramakrishnan et al. [6] who introduced clean-up jobs to reduce the memory footprint and propose some simple heuristics. Their work was continued by Bharathi et al. [16] who developed genetic algorithms to schedule such workflows. More recently, runtime schedulers have also been confronted to the problem: in StarPU, attempts have been made to reduce memory consumption by throttling the task submission rate [17].

As explained in the introduction, our study extends the work of Sbîrlea at al. [8]. 
This study focuses on a different model, in which all data have the same size (as for register allocation). They target smaller-grain tasks in the Concurrent Collections (CnC) programming model [18], a stream/dataflow programming language. Their objective is, as ours, to schedule a DAG of tasks using a limited memory. For this, they associate a color to each memory slot and then build a coloring of the data, in which two data items with the same color cannot coexist. If the number of colors is not sufficient, additional dependence edges are introduced to prevent too many data items to coexist. These additional edges respect a pre-computed sequential schedule to ensure acyclicity. An extension to support data of different sizes is proposed, which conceptually allocates several colors to a single data, but is only suitable for a few distinct sizes. Note that the idea of restricting the memory need of a computation by adding edges to a graph has also been used in [19, Chapter 4] to limit register usage with Instruction Level Parallelism.

Compared to the existing work, the present work studies graphs with arbitrary data sizes, and it formally defines the problem of transforming a graph to cope with a strong memory bound: this allows the use of efficient dynamic scheduling heuristics at runtime with the guarantee to never exceed the memory bound.

\section{Problem modeling}

\subsection{Formal description}

As stated before, we consider that the targeted application is described by a workflow of tasks whose precedence constraints form a DAG $G=(V, E)$. Its nodes $i \in V$ represent tasks and its edges $e \in E$ represent precedence relations, in the form

of input and output data. The processing time necessary to complete a task $i \in V$ is denoted by $w_{i}$. In our model, the memory usage of the computation is modeled only by the size of the data produced by the tasks and represented by the edges. Therefore, for each edge $e=(i, j)$, we denote by $m_{e}$ or $m_{i, j}$ the size of the data produced by task $i$ for task $j$. We assume that $G$ contains a single source node $s$ and a single sink node $t$; otherwise, one can add such nodes along with the appropriate edges, all of null weight. An example of such a graph is illustrated in Figure 1. For the sake 
of simplicity, we define the following sizes of inputs and outputs of a node $i$ :

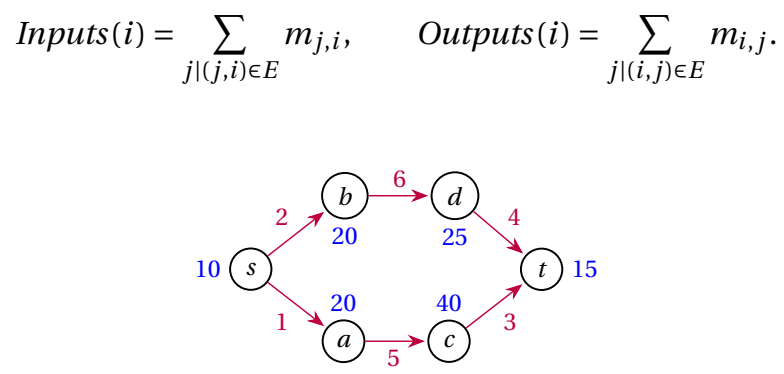

Figure 1: Example of a workflow, (red) edge labels represent the size $m_{i, j}$ of associated data, while (blue) node labels represent their computation weight $w_{i}$.

We propose here to use a very simple memory model, which might first seem unrealistic, but it will indeed prove itself very powerful both to model complex memory behaviors and to express the peak memory usage. In the proposed model, at the beginning of the execution of a task $i$, all input data of $i$ are immediately deleted from the memory, while all its output data are allocated to the memory. That is, the total amount of memory $M_{\text {used }}$ needed to store all necessary data is transformed as follows:

$$
M_{\mathrm{used}} \leftarrow M_{\mathrm{used}}-\operatorname{Inputs}(i)+\operatorname{Outputs}(i) .
$$

This model, called the SimpleDataFlowModel, is extremely simple, and in particular does not allow a task to have both its inputs and outputs simultaneously in memory. However, we will see right below that it is expressive enough to emulate other complex and more realistic behaviors.

Before considering other memory models, we start by defining some terms and by comparing sequential schedules and parallel executions of the graph. We say that the data associated to the edge $(i, j)$ is active at a given time if the execution of $i$ has started but not the one of $j$. This means that this data is present in memory. A sequential schedule $\mathscr{S}$ of a DAG $G$ is defined by an order $\sigma$ of its tasks. The memory used by a sequential schedule at a given time is the sum of the sizes of the active data. The peak memory of such a schedule is the maximum memory used during 
its execution, which is given by:

$$
M_{\text {peak }}(\sigma)=\max _{i} \sum_{j \text { s.t. } \sigma(j) \leq \sigma(i)} \operatorname{Outputs}(j)-\operatorname{Inputs}(j)
$$

where the set $\{j$ s.t. $\sigma(j) \leq \sigma(i)\}$ represents the set of tasks started before task $i$, including itself.

A parallel execution of a graph on $p$ processors is defined by:

- An allocation $\mu$ of the tasks onto the processors (task $i$ is computed on processor $\mu(i)$ );

- The starting times $\sigma$ of the tasks (task $i$ starts at time $\sigma(i)$ ).

As usual, a valid schedule ensures that data dependences are satisfied $(\sigma(j) \geq \sigma(i)+$ $w_{i}$ whenever $(i, j) \in E$ ) and that processors compute a single task at each time step (if $\mu(i)=\mu(j)$, then $\sigma(j) \geq \sigma(i)+w_{i}$ or $\left.\sigma(i) \geq \sigma(j)+w_{j}\right)$. Note that when considering parallel executions, we assume that all processors use the same shared memory, whose size is limited.

A very important feature of the proposed SIMPLEDATAFLOWMODEL is that there is no difference between sequential schedules and parallel executions as far as memory is concerned, which is formally stated in the following theorem.

Theorem 1. For each parallel execution $(\mu, \sigma)$ of a DAG $G$, there exists a sequential schedule with equal peak memory.

Proof. We consider such a parallel execution, and we build the corresponding sequential schedule by ordering tasks in non decreasing starting time. Since in the SimpleDATAFlOWMODEL, there is no difference in memory between a task being processed and a completed task, the sequential schedule has the same amount of used memory as the parallel execution after the beginning of each task. Thus, they have the same peak memory.

This feature will be very helpful when computing the maximum memory of any parallell execution, in Section 4: thanks to the previous result, it is equivalent to computing the peak memory of a sequential schedule. 


\subsection{Emulation of other memory models}

\subsubsection{Classical workflow model}

As we explained above, our model does not allow inputs and outputs of a given task to be in memory simultaneously. However, this is a common behavior, and some studies, such as [20], even consider that in addition to inputs and outputs, some temporary data $m_{i}^{\text {temp }}$ has to be in memory when processing task $i$. The memory needed for its processing is then Inputs $(i)+m_{i}^{\text {temp }}+\operatorname{Outputs}(i)$. Although this is very different to what happens in the proposed SIMPLEDATAFLOWMODEL, such a behavior can be simply emulated, as illustrated on Figure 2. For each task $i$, we split it into two nodes $i_{1}$ and $i_{2}$. We transform all edges $(i, j)$ in edges $\left(i_{2}, j\right)$, and edges $(k, i)$ in edges $\left(k, i_{1}\right)$. We also add an edge $\left(i_{1}, i_{2}\right)$ with an associated data of size $\operatorname{Inputs}(i)+m_{i}^{\text {temp }}+\operatorname{Outputs}(i)$. Task $i_{1}$ represents the allocation of the data needed for the computation, as well as the computation itself, and its work is thus $w_{i_{i}}=w_{i}$. Task $i_{2}$ stands for the deallocation of the input and temporary data and has work $w_{i_{2}}=0$.

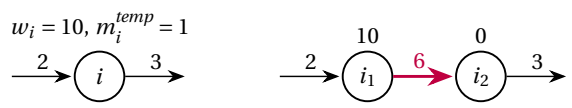

Figure 2: Transformation of a task as in [20] (left) to the SimPLEDATAFLOWMODEL (right).

\subsubsection{Shared output data}

Our model considers that each task produces a separate data item for each of its successors. However, it may well happen that a task $i$ produces an output data $d$, of size $m_{i, d}^{\text {shared }}$, which is then used by several of its successors, and is freed after the completion of its last successor. The output data is then shared among successors, contrarily to what is considered in the SiMPLEDATAFLOWMODEL. Any task can then produce several output data, some of which can be shared among several successors. Again, such a behavior can be emulated in the proposed model, as illustrated on Figure 3.

Such a task $i$ with a shared output data will first be transformed as follows. For

each shared output data $d$ of size $m_{i, d}^{\text {shared }}$, we add a task $i_{d}$ which represents the 

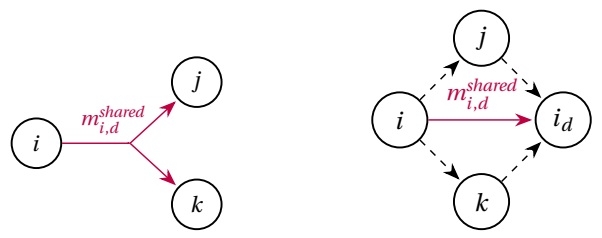

Figure 3: Transformation of a task $i$ with a single shared output data into SIMPLEDATAFLOWMODEL. The plain (red) edge carries the shared data size, while dashed (black) edges have null size.

deallocation of the shared data $d$ (and thus has null computation time $w_{i_{d}}$ ). An edge of size $m_{i, d}^{\text {shared }}$ is added between $i$ and the deallocation task $i_{d}: m_{i, i_{d}}=m_{i, d}^{\text {shared }}$. Data dependence to a successor $j$ sharing the output data $d$ is represented by an edge $(i, j)$ with null data size $\left(m_{i, j}=0\right)$ (if it does not already exist, due to an other data produced by $i$ and consumed by $j$ ). Finally, for each such successor $j$, we add an edge of null size $\left(j, i_{d}\right)$ to ensure that the shared data will be freed only when it has been used by all the successors sharing it. The following result states that after this transformation, the resulting graph exhibits a similar memory behavior.

Theorem 2. Let $G$ be a DAG with shared output data, and $G^{\prime}$ its transformation into SIMPLEDATAFLOWMODEL. If there exists a schedule $\mathscr{S}$ of $G$ with peak memory $M$, then there exists a schedule $\mathscr{S}^{\prime}$ of $G^{\prime}$ with peak memory $M$. Conversely, if there exists a schedule $\mathscr{S}^{\prime}$ of $G^{\prime}$ with peak memory $M^{\prime}$, then there exists a schedule $\mathscr{S}$ of $G$ with peak memory $M \leq M^{\prime}$.

The proof of this result is not difficult but technical and is therefore deferred to the companion research report [21].

Note that there may exist a schedule $\mathscr{S}^{\prime}$ of the transformed graph $G^{\prime}$ with peak larger than $M$, the largest memory peak of any schedule $\mathscr{S}$ of the original graph $G$, as it is possible to schedule all deallocation vertices $i_{d}$ at the very end of the schedule. Then, all shared data stays in memory until the very end of the computation, which is not realistic. Hence, the computation of the maximum memory peak has a different complexity when using shared data, as highlighted in Section 4.3. However, when considering memory minimization, which is the main goal in this paper, deallocation vertices $i_{d}$ must be processed as soon as possible, which corresponds 
to what happens with shared data.

\subsubsection{Pebble game}

One of the pioneer work dealing with the memory footprint of a DAG execution has been conducted by Sethi [22]. He considered what is now recognized as a variant of the PebbleGame model. We now show that the proposed SimpleDataFlowModel is an extension of PebbleGame. The pebble game is defined on a DAG as follows:

- A pebble can be placed at any time on a node with no predecessor;

- A pebble can be placed on a node if all its predecessors have a pebble;

- A pebble can be removed from a node at any time;

- A pebble cannot be placed on a node that has been previously pebbled.

The objective is to pebble all the nodes of a given graph, using a minimum number of pebbles. Note that the pebble of a node should be removed only when all its successors are pebbled. This is the main difference with our model, where a node produces a different output data for each of its successors. Thus, the PebbleGame model ressembles the model with shared output data presented above, with all data of size one. We thus apply the same transformation and consider that a pebble is a shared output data used for all the successors of a node. In addition, we add a fictitious successor to all nodes without successors. Hence, the pebble placed on such a node can be considered as the data consumed by this successor. Then, we are able to prove that the memory behavior of the transformed graph under SIMPLEDATAFLOWMODEL corresponds to the pebbling of the original graph, as outlined by the following theorem.

Theorem 3. Let $P$ be a DAG representing an instance of a PEBBLEGAME problem, and $G$ its transformation into SIMPLEDATAFLOWMODEL. There exists a pebbling scheme $\mathscr{P}$ of P using at most B pebbles if and only if there exists a schedule $\mathscr{S}^{\prime}$ of $G^{\prime}$ with peak memory at most $B$.

As for Theorem 2, the proof of this result is deferred to the research report [21]. 


\subsection{Peak memory minimization in the proposed model}

The emulation of the PeBbleGame problem, as proposed above, allows us to formally state the complexity of minimizing the memory of a DAG, as expressed by the following theorem.

Theorem 4. Deciding whether an instance of SimpleDATAFLOWMODEL can be scheduled with a memory of limited size is NP-complete.

Proof. The problem of deciding whether an instance of PEBBLEGAME can be traversed with a given number of pebbles is NP-complete [22]. Then, thanks to Theorem 3, we know that an instance of PEBBLEGAME can be transformed into an instance of SiMPLEDATAFLOWMODEL (with twice as many nodes), which then inherits of this complexity result.

\section{Computing the maximal peak memory}

In this section, we are interested in computing the maximal peak memory of a given DAG $G=(V, E)$, that is, the largest peak memory that can be reached by a sequential schedule of $G$. Our objective is to check whether a graph can be safely executed by a dynamic scheduler without exceeding the memory bound. We first focus on the model presented in Section 3.1. Then, in Section 4.3, we study the case of shared data.

We first define the notion of topological cut. We recall that $G$ contains a single source node $s$ and a single sink node $t$.

Definition 1. A topological cut $(S, T)$ of a DAGG is a partition of $\mathrm{B}$ in two sets of nodes $S$ and $T$ such that $s \in S, t \in T$, and no edge is directed from a node of $T$ to a node of $S$. An edge $(i, j)$ belongs to the cut if $i \in S$ and $j \in T$. The weight of a topological cut is the sum of the weights of the edges belonging to the cut.

For instance, in the graph of Figure 1 , the cut $(\{s, a, b\},\{c, d, t\})$ is a topological cut of weight 11 . Note that this cut would not be a topological cut if the edge $(d, a)$ was present in the graph. In the SIMPLEDATAFLOWMODEL, the memory used at a given time is equal to the sum of the sizes of the active output data, which depends solely 
on the set of nodes that have been executed or initiated. Therefore, the maximal peak memory of a DAG is equal to the maximum weight of a topological cut.

Definition 2. The MAXTOPCUT problem consists in computing a topological cut of maximum weight for a given DAG.

We first prove that this problem is polynomial, by providing a linear program over the rationals solving it, and then propose an explicit algorithm which does not rely on linear programming.

\subsection{Complexity of the problem}

The MaXTopCuT problem belongs to the family of problems in which we are interested in computing a weighted cut in a graph that optimizes some quantity.

The problem of finding a cut of minimum weight (when edge weights are nonnegative) has been thoroughly studied in the literature, and many polynomialtime algorithms have been proposed to solve it, both for undirected and directed graphs [23]. On the opposite, computing a maximal cut is in general much more difficult. It is well-known that this problem is NP-complete on general graphs, either undirected or directed [24], and with unit weights [25]. In 2011, Lampis et al. even extended this result to DAGs [26], which are our scope of interest. However, our problem is more restrictive, as we are only interested in maximal topological cuts on DAGs, which means that all the edges of the cut have the same direction. As illustrated above on Figure 1, this constraint heavily reduces the set of possible cuts. There are $2^{n}$ possible cuts for any DAG with $n$ nodes: the number of ways to partition the nodes in two sets. However, the number of topological cuts can be much

lower: only $n-1$ possibilities for a chain graph on $n$ nodes. The problem of finding a maximal topological cut is then intuitively easier than finding a maximal cut in a DAG.

We show that MAXTOPCUT is actually polynomial by exhibiting a Linear Program solving it. This proof is adapted from [27].

Theorem 5. The problem of finding a maximal topological cut in a DAG is polynomial. 
Proof. We consider a DAG $G$, where each edge $(i, j)$ has a weight $m_{i, j}$.

We now consider the following linear program $\mathscr{P}$ :

$$
\begin{aligned}
\max \sum_{(i, j) \in E} m_{i, j} d_{i, j}, \quad \text { s.t. } \\
\forall(i, j) \in E, \quad d_{i, j}=p_{i}-p_{j}, \\
\forall(i, j) \in E, \quad d_{i, j} \geq 0 \\
p_{s}=1, \\
p_{t}=0 .
\end{aligned}
$$

Intuitively, an integer solution of $\mathscr{P}$ corresponds to a valid topological cut $(S, T)$. The variable $p_{i}$ represents the potential of vertex $i$ : if it is equal to 1 then $i \in S$ and if it is equal to 0 then $i \in T$. Then, $d_{i, j}$ is equal to 1 if the edge $(i, j)$ belongs to the cut $(S, T)$ and 0 otherwise. Finally, the objective function represents the weight of the cut. However, a general solution of $\mathscr{P}$ consists of rational numbers and not integers, so does not correspond directly to a topological cut. Nevertheless, we show that for this particular program, a naive rounding algorithm exhibits a topological cut, which can then be computed in polynomial time.

Note that $\mathscr{P}$ is similar to the classic linear program computing the minimal $s-t$ cut [23]. The only differences are Equation (2) being an equality instead of an inequality, and the direction of the objective function.

We begin by proving that if $G$ admits a topological cut of weight $M$, there is a solution of the linear program for which the objective function equals $M$. Let $(S, T)$ be a topological cut of $G$. For every node $i$, we define $p_{i}=1$ if $i \in S$ and $p_{i}=0$ if $i \in T$. Then, for each edge $(i, j)$ belonging to the cut, we have $p_{i}-p_{j}=1$ and for the remaining edges $(i, j)$, we have $p_{i}-p_{j}=0$. Indeed, no edge can be directed from $T$ to $S$ by definition. Therefore, we have for all $(i, j) \in E, d_{i, j}=p_{i}-p_{j} \geq 0$ so the proposed valuation satisfies $\mathscr{P}$, and the objective function is equal to the weight of $(S, T)$.

Now, suppose that $\mathscr{P}$ admits a valid rational solution of objective function $M^{*}$. We prove that there exists a topological cut $\left(S^{*}, T^{*}\right)$ of $G$ of weight at least $M^{*}$. Note 
that for any edge $(i, j)$, we have $d_{i, j} \geq 0$ so $p_{i} \geq p_{j}$. Then, every node of $G$ belongs to a directed path from $s$ to $t$ by definition of $s$ and $t$. Therefore, every $p_{i}$ belongs to [0,1]. Indeed, for a given $i \in V$, let $v_{1}, v_{2}, \ldots, v_{k}$ be the vertices of a directed path from $i$ to $t$, with $i=v_{1}$ and $t=v_{k}$. Then, we deduce that $p_{i}=p_{\nu_{1}} \geq p_{v_{2}} \geq \cdots \geq p_{v_{k}}=p_{t}=0$. A similar proof with a path from $s$ to $i$ shows that $p_{i}$ is not larger than 1 .

In order to prove the existence of $\left(S^{*}, T^{*}\right)$, we consider a random topological cut $(S, T)$ defined as follows: draw $r$ uniformly in ]0, 1 [, and let $(S, T)$ be the cut $\left(S_{r}, T_{r}\right)$, with $S_{r}=\left\{i \mid p_{i}>r\right\}$ and $T_{r}=\left\{j \mid p_{j} \leq r\right\}$. This partition is valid as, for any $i \in S_{r}$ and $j \in T_{r}$, we have $p_{i}>p_{j}$, so the edge $(j, i)$ cannot belong to $E$ : this would imply $d_{j, i}<0$ which violates a constraint of $\mathscr{P}$. Now, let us compute the expected weight $M(S, T)$ of the cut $(S, T)$. The probability for a given edge $(i, j)$ to belong to $(S, T)$ is exactly $d_{i, j}=p_{i}-p_{j}$, as $r$ is drawn uniformly in $] 0,1$ [ and all $p_{i}$ belong to $[0,1]$. Therefore, the expected cost of $(S, T)$ is given by

$$
\begin{aligned}
E(M(S, T)) & =\sum_{(i, j) \in E} m_{i, j} \operatorname{Pr}((i, j) \text { belongs to }(S, T)) \\
& =\sum_{(i, j) \in E} m_{i, j} d_{i, j}=M^{*} .
\end{aligned}
$$

Therefore, there exists $r \in] 0,1\left[\right.$ such that $M\left(S_{r}, T_{r}\right) \geq M^{*}$, which proves the existence of a topological cut $\left(S^{*}, T^{*}\right)$ of weight at least $M^{*}$. Note that an algorithm could then find such a topological cut by computing $M\left(S_{p_{i}}, T_{p_{i}}\right)$ for every $i \in V$.

We now show that it is not necessary, as, if $M^{*}$ is the optimal objective value, then the weight of any cut $\left(S_{r}, T_{r}\right)$ is equal to $M^{*}$. Note that no cut $\left(S_{r}, T_{r}\right)$ can have a weight larger than $M^{*}$ by definition. So, for all $r$, we have $M\left(S_{r}, T_{r}\right) \leq M^{*}$. As $E(M(S, T))=M^{*}$, we conclude that $\left.\operatorname{Pr}\left(M(S, T)<M^{*}\right)\right)=0$. It remains to show that no single value of $r$ can lead to a suboptimal cut. Assume by contradiction that there exists $\left.r_{0} \in\right] 0,1\left[\right.$ such that $M\left(S_{r_{0}}, T_{r_{0}}\right)<M^{*}$. Let $r_{1}=\min \left\{p_{i} \mid p_{i}>r_{0}\right\}$, which is defined as $p_{t}=1>r_{0}$, and consider any $r \in\left[r_{0}, r_{1}\right.$ [. For every $i \in V$, if $p_{i}>r_{0}$ then $p_{i} \geq r_{1}>r$, and if $p_{i} \leq r_{0}$ then $p_{i} \leq r$, so, by definition of $S_{r}$ and $T_{r}$, we have $\left(S_{r}, T_{r}\right)=\left(S_{r_{0}}, T_{r_{0}}\right)$. Therefore, we get

$$
\left.\operatorname{Pr}\left(M(S, T)<M^{*}\right)\right) \geq \operatorname{Pr}\left((S, T)=\left(S_{r_{0}}, T_{r_{0}}\right)\right) \geq r_{1}-r_{0}>0 .
$$

This inequality contradicts the fact that $\left.\operatorname{Pr}\left(M(S, T)<M^{*}\right)\right)=0$. 
To conclude, a maximal topological cut can be computed by first solving the linear program $\mathscr{P}$ in rationals, then selecting any cut $\left(S_{r}, T_{r}\right)$, for instance by taking $r=1 / 2$.

\subsection{Explicit algorithm}

In the previous section, we have exhibited a linear program solving the MAXTOPCUT problem. We are now interested in an explicit polynomial algorithm, which allows us to have a different approach on the problem, and to solve it without relying on a linear program solver. We first consider a problem related to the dual version of MAXTOPCUT, which we call MinFLow:

Definition 3. The MinFlow problem consists in computing a flow of minimum value where the amount of flow that passes through an edge is not smaller than the edge weight.

We recall that the value of a flow $f$ is defined as $\sum_{j,(s, j) \in E} f_{s, j}$. In this problem the edge weights do not represent capacities as in a traditional flow, but rather demands: the minimum flow must be at least as large as these demands on all edges ${ }^{1}$. We recall that the MAXFLOW problem consists in finding a flow of maximum value where the amount of flow that passes through each edge is not larger than the edge weight. Its dual version, the MinCUT problem, consists in computing the st-cut $(S, T)$ of minimum weight, where $s \in S$ and $t \in T$. Note that this cut may not be topological. See [28, Chapter 26] for more details. The MinFLow problem is described by the following linear program.

$$
\begin{gathered}
\min \sum_{j \mid} \sum_{(s, j) \in E} f_{s, j}, \quad \text { s.t. } \\
\forall j \in V \backslash\{s, t\}, \quad\left(\sum_{i \mid(i, j) \in E} f_{i, j}\right)-\left(\sum_{k \mid(j, k) \in E} f_{j, k}\right)=0, \\
\forall(i, j) \in E, \quad f_{i, j} \geq m_{i, j} .
\end{gathered}
$$

\footnotetext{
${ }^{1}$ This must not be mistaken with the demands of vertices (i.e., the value of the consumed flow) as in the Minimum Cost Flow problem.
} 
We propose in Algorithm 1 an explicit algorithm to resolve the MAXTOPCUT problem. A similar algorithm for a very close problem has been proposed in [29]. We first need an upper bound $f_{\text {max }}$ on the value of the optimal flow solving the dual Minflow problem on $G$. We can take for instance $f_{\max }=1+\sum_{(i, j) \in E} m_{i, j}$. The algorithm builds a flow $f$ with a value at least $f_{\text {max }}$ on all edges. Intuitively, the flow $f$ can be seen as an optimal flow $f^{*}$ solving the MinFLOw problem, on which has been added an arbitrary flow $f^{+}$. In order to compute $f^{*}$ from $f$, the algorithm explicitly computes $f^{+}$, by solving a MAXFLOW instance on a graph $G^{+}$. Intuitively, this step consists in maximizing the flow that can be subtracted from $f^{*}$. Finally, the maximum topological cut associated to the flow $f^{*}$ is actually equal to the minimum st-cut of $G^{+}$that can be deduced from the residual network induced by $f^{+}$. We recall that the residual network of $G^{+}$induced by $f^{+}$contains each edge $(i, j)$ such that either $(i, j) \in E$ and $f_{i, j}^{+}<m_{i, j}^{+}$or $(j, i) \in E$ and $f_{j, i}^{+}>0$, as defined for instance in [29].

The complexity of Algorithm 1 depends on two implementations: how we compute the first flow $f$ and how we solve the MAXFLOW problem. The rest is linear in the number of edges. Computing the starting flow $f$ can be done by looping over all edges, finding a simple path from $s$ to $t$ containing a given edge, and adding a flow going through that path of value $f_{\max }$. Note that this method succeeds because the graph is acyclic, so every edge is part of a simple path (without cycle) from $s$ to $t$. This can be done in $O(|V||E|)$. Solving the MAXFLow problem can de done in $O\left(|V||E| \log \left(|V|^{2} /|E|\right)\right)$ using Goldberg and Tarjan's algorithm [30]. Therefore, Algorithm 1 can be executed in time $O\left(|V||E| \log \left(|V|^{2} /|E|\right)\right)$.

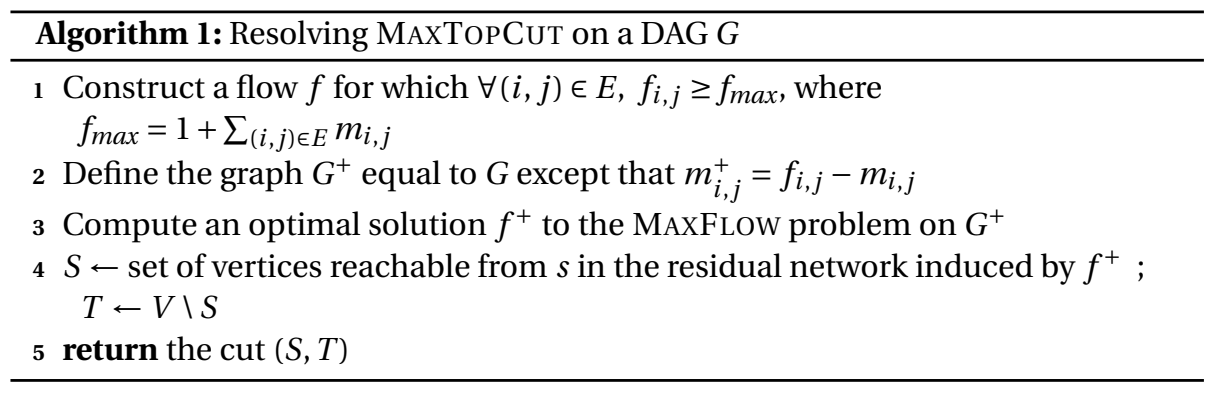


Theorem 6. Algorithm 1 solves the MAXTOPCUT problem.

Proof. We start by showing that the cut $(S, T)$ is a topological cut. We have $s \in S$ and $t \in T$ by definition. We now show that no edge exist from $T$ to $S$ in $G$. By definition of $S$, no edge exist from $S$ to $T$ in the residual network, so if there exists an edge $(j, i)$ from $T$ to $S$ in $G$, it verifies $f_{j, i}^{+}=0$. We then show that every edge of $G$ has a positive flow going through it in $f^{+}$, which proves that there is no edge from $T$ to $S$.

Assume by contradiction that there exists an edge $(k, \ell)$ such that $f_{k, \ell}^{+}$is null. Let $S_{k} \subset V$ be the set of ancestors of $k$, including $k$. Then, $S_{k}$ contains $s$ but not $t$ nor $\ell$ as $G$ is acyclic. Denoting $T_{k}=V \backslash S_{k}$, we get that $\left(S_{k}, T_{k}\right)$ is a topological cut as no edge goes from $T_{k}$ to $S_{k}$ by definition. The weight of the cut $\left(S_{k}, T_{k}\right)$ is at most the value of the flow $f$, which is $|f|$. As $f_{k, \ell}^{+}=0$, the amount of flow $f^{+}$that goes through this cut is at most $|f|-f_{k, \ell} \leq|f|-f_{\text {max }}$. Therefore, the value of $f^{+}$verifies $\left|f^{+}\right| \leq|f|-f_{\text {max }}$.

Now, we exhibit a contradiction by computing the amount of flow $f^{+}$passing through the cut $(S, T)$. By definition of $(S, T)$, all the edges from $S$ to $T$ are saturated in the flow $f^{+}$: for each edge $(i, j) \in E$ with $i \in S$ and $j \in T$, we have $f_{i, j}^{+}=m_{i, j}^{+}=$ $f_{i, j}-m_{i, j}$. The value of the flow $f^{+}$is equal to the amount of flow going from $S$ to $T$ minus the amount going from $T$ to $S$. Let $E_{S, T}$ (resp. $E_{T, S}$ ) be the set of edges between $S$ and $T$ (resp. $T$ and $S$ ). We have the following (in)equalities:

$$
\begin{aligned}
\left|f^{+}\right| & =\left(\sum_{(i, j) \in E_{S, T}} f_{i, j}^{+}\right)-\left(\sum_{(j, i) \in E_{T, S}} f_{j, i}^{+}\right) \\
& \geq\left(\sum_{(i, j) \in E_{S, T}}\left(f_{i, j}-m_{i, j}\right)\right)-\left(\sum_{(j, i) \in E_{T, S}} f_{j, i}\right) \\
& \geq|f|-\left(\sum_{(i, j) \in E_{S, T}} m_{i, j}\right)>|f|-f_{\max } .
\end{aligned}
$$

Therefore, we have a contradiction on the value of $\left|f^{+}\right|$, so no edge exists from $T$ to $S$ and $(S, T)$ is a topological cut.

Now, we define the flow $f^{*}$ on $G$, defined by $f_{i, j}^{*}=f_{i, j}-f_{i, j}^{+} \geq m_{i, j}$. We show that $f^{*}$ is an optimal solution to the MinFLOW problem on $G$. It is by definition a valid solution as $f_{i, j}^{+} \leq m_{i, j}^{+}=f_{i, j}-m_{i, j}$ so $f_{i, j}^{*}=f_{i, j}-f_{i, j}^{+} \geq f_{i, j}+m_{i, j}-f_{i, j}=m_{i, j}$. Let $g^{*}$ be an optimal solution to the MinFLOW problem on $G$ and $g^{+}$be the flow defined 
by $g_{i, j}^{+}=f_{i, j}-g_{i, j}^{*}$. By definition, $g_{i, j}^{*} \geq m_{i, j}$ so $g_{i, j}^{+} \leq f_{i, j}-m_{i, j}=m_{i, j}^{+}$. Furthermore, we know that $g_{i, j}^{*} \leq f_{\max }$ because there exists a flow, valid solution of the MinFLow problem, of value $\sum_{(i, j) \in E} m_{i, j} \leq f_{\max }$ : simply add for each edge $(i, j)$ a flow of value $m_{i, j}$ passing through a path from $s$ to $t$ containing the edge $(i, j)$. Then, we have $g_{i, j}^{*} \leq f_{\max } \leq f_{i, j}$ so $g_{i, j}^{+} \geq 0$ and $g^{+}$is therefore a valid solution of the MAXFLow problem on $G^{+}$, but not necessarily optimal.

So the value of $g^{+}$is not larger than the value of $f^{+}$by optimality of $f^{+}$, and therefore, the value of $f^{*}$ is not larger than the value of $g^{*}$. Finally, $f^{*}$ is an optimal solution to the MinFLOW problem on $G$.

Now, we show that $(S, T)$ is a topological cut of maximum weight in $G$. Let $\left(S_{0}, T_{0}\right)$ be any topological cut of $G$. The total amount of flow of $f^{*}$ passing through the edges belonging to $\left(S_{0}, T_{0}\right)$ is equal to the value of $f^{*}$. As for all $(i, j) \in E$ we have $f_{i, j}^{*} \geq m_{i, j}$, the weight of the cut $\left(S_{0}, T_{0}\right)$ is not larger than the value of $f^{*}$. It remains to show that this upper bound is reached for the cut $(S, T)$. By the definition of $(S, T)$, we know that for $(i, j) \in(S, T)$, we have $f_{i, j}^{+}=m_{i, j}^{+}=f_{i, j}-m_{i, j}$. Therefore, on all these edges, we have $f_{i, j}^{*}=f_{i, j}-f_{i, j}^{+}=m_{i, j}$, so the value of the flow $f^{*}$ is equal to the weight of $(S, T)$.

Therefore, $(S, T)$ is an optimal topological cut.

\subsection{Maximal peak memory with shared data}

In this section, we focus on the model allowing shared data, as proposed in Section 3.2.2: a task $i$ may produce a data which is used by multiple successors $j_{1}, \ldots, j_{k}$. This data is kept in memory until all successors are completed, and deleted when the last successor is completely processed. Surprisingly, using such shared data largely complexifies the problem. Note that, as stated in Section 3.2.2, the transformation to the SIMPLEDATAFLOWMODEL overestimates the maximal peak memory. This intuitively explains why the complexity of computing maximal peak memory with shared data is different from the complexity of the same problem in SIMPLEDataFlowModel. Nevertheless, as the main objective of the paper is to lower the maximum peak memory when processing a task graph, having an upper bound on this peak memory is sufficient: we will aim at limiting the maximum peak memory 
of the transformed graph without shared data, which guarantees that the maximum peak memory of the original graph is also below the prescribed bound.

The following result is inspired and adapted from the work of Touati [19, 31] which studies the maximum number of registers that may be needed by the execution of a DAG of instructions on a superscalar processor. The model (and thus the proof) is quite different, but the conclusion is the same.

Theorem 7. Given a DAG G with shared data and a memory bound $M$, the problem of deciding whether or not there is a sequential schedule of $G$ which uses a memory larger than, or equal to M is NP-Complete.

Proof. Given a sequential schedule, it is easy to simulate it and to check whether the maximum peak memory is greater than or equal to $M$; hence, the problem belongs to NP.

To prove the completeness, we consider the NP-complete set cover problem (or minimum cover problem, SP5 in [32]). It consists of a set $S$ and a collection $\mathscr{C}=$ $\left\{C_{1}, C_{2}, \ldots\right\}$ of subsets of $S$, and a positive integer $k \leq|\mathscr{C}|$. The problem consists in finding in $\mathscr{C}$ a cover of size $k$ or less, that is, a subset $\mathscr{C}^{\prime} \subseteq \mathscr{C}$ with $\left|\mathscr{C}^{\prime}\right| \leq k$ such that every element of $S$ belongs to at least a member of $\mathscr{C}^{\prime} . \mathscr{C}$ is assumed to be a cover of size $|\mathscr{C}|$. For readability, we note $n=|S|$ and $m=|\mathscr{C}|$.

We consider an instance of the set cover problem. We first assume that $n \geq m$. Otherwise, we add $m-n+1$ elements in $S$ and a single subset in $\mathscr{C}$ which covers exactly all new elements. We build an instance of the maximum peak memory problem with shared data. Its graph $G$ contains the following vertices:

- $A_{1}, \ldots, A_{n}$, which do not have any predecessors, and produce each a single output of size 1;

- $B_{1}, \ldots, B_{m}$, the predecessors of $B_{i}$ are the $A_{j}$ 's such that $s_{j} \in \mathscr{C}_{i}$, and each $B_{i}$ produces a single output data of size 1 ;

- A target vertex $T$, whose predecessors are all the $B_{i}$ 's vertices.

Thus, the output of $A_{j}$ is shared by all the $B_{i}$ vertices such that $s_{j}$ is covered by $C_{i}$. The proof relies on the following lemma. 
Lemma 1. There exists in $\mathscr{C}$ a cover of size at most $k$ if and only if there exists a schedule of $G$ with peak memory at least $n+m-k$.

If this lemma is true, the NP-completeness of the maximum peak memory with shared data naturally stems from the NP-completeness of the set cover problem. We now prove the lemma.

First, we consider a solution $\mathscr{C}^{\prime}$ of the cover problem with $\left|\mathscr{C}^{\prime}\right| \leq k$. We build a schedule of $G$ as follows:

1. We first schedule all $A_{j}$ vertices, in any order.

2. We then schedule all $B_{i}$ vertices with $i \notin \mathscr{C}^{\prime}$, in any order.

3. We then schedule all remaining $B_{i}$ vertices, and finally vertex $T$.

Note that after Step 2, the output of all $A_{j}$ vertices must still reside in memory, as each of them is used by some $B_{i}$ for $i \in \mathscr{C}^{\prime}$, since $\mathscr{C}^{\prime}$ covers all elements of $S$. Hence, the memory used after Step 2 is $n+\left(m-\left|\mathscr{C}^{\prime}\right|\right) \geq n+(m-k)$. The peak memory of this schedule is thus not smaller than $n+m-k$.

Second, we consider a schedule with maximum peak memory $M \geq n+m-k$. We first show how to transform the schedule so that the outputs of all the $A_{j}$ 's are in memory when the peak $M$ is reached. Let $\mathscr{A}$ (respectively $\mathscr{B}$ ) be the set of the $A_{j}$ 's (resp. the $B_{i}$ 's) whose output are in memory when the peak is (first) reached. Assume that some $A_{j}$ is not in $\mathscr{A}$. Then, there exists at least one $B_{i} \in \mathscr{B}$ which is a successor of $A_{j}$ (otherwise the output of $A_{j}$ would still be in memory). We build $\mathscr{A}^{\prime}$ by adding $A_{j}$ in $\mathscr{A}$ as well as all other predecessors of $B_{i}$ that are not in $\mathscr{A}$. We also build $\mathscr{B}^{\prime}$ by removing $B_{i}$ from $\mathscr{B}$. We consider the schedule that first processes all the $A_{j}$ 's, then the nodes of $\mathscr{B}^{\prime}$. At this moment, the outputs of all the nodes of $\mathscr{A}^{\prime}$ are in memory, including $A_{j}$, and the memory used is $\left|\mathscr{A}^{\prime}\right|+\left|\mathscr{B}^{\prime}\right|$ which is at least $M$ as $\left|\mathscr{A}^{\prime}\right| \geq|\mathscr{A}|+1$ and $\left|\mathscr{B}^{\prime}\right|=|\mathscr{B}|-1$ and $|\mathscr{A}|+|\mathscr{B}|=M$. By repeating this process, we obtain a schedule with peak memory at least $n+m-k$ which reaches the peak with the outputs of all the $A_{j}$ 's in memory. Thus, at least $m-k$ of the $B_{i}$ outputs are in memory during the peak. The remaining $B_{i}$ vertices, whose number is at most $k$, correspond to a cover of $S$. 


\section{Lowering the maximal peak memory}

We now move back to the case where no shared data is used. In Section 4, we have proposed a method to determine the maximal topological cut of a DAG, which is equal to the maximal peak memory of any (sequential or parallel) traversal. We now move to the problem of scheduling such a graph within a bounded memory $M$. If the maximal topological cut is at most $M$, then any schedule of the graph can be executed without exceeding the memory bound. Otherwise, it is possible that we fail to schedule the graph within the available memory. One solution would be to provide a complete schedule of the graph onto a number $p$ of computing resources, which never exceeds the memory. However, using a static schedule can lead to very poor performance if the task duration are even slightly inaccurate, or if communication times are difficult to predict, which is common on modern computing platforms. Hence, our objective is to let the runtime system dynamically choose the allocation and the precise schedule of the tasks, but to restrict its choices to avoid memory overflow.

In this section, we solve this problem by transforming a graph so that its maximal peak memory becomes at most $M$. Specifically, we aim at adding some new edges to $G$ to limit the maximal topological cut. Consider for example the toy example of Figure 1. Its maximal topological cut has weight 11 and corresponds to the output data of tasks $a$ and $b$ being in memory. If the available memory is only $M=10$, one may for example add an edge $(d, a)$ of null weight to the graph, which would result in a maximal topological cut of weight 9 (output data of $a$ and $d$ ). Note that on this toy example, adding this edge completely serializes the graph: the only possible schedule of the modified graph is sequential. However, this is not the case of realistic, wider graphs. We formally define the problem as follows.

Definition 4. A partial serialization of a $D A G G=(V, E)$ for a memory bound $M$ is a DAG $G^{\prime}=\left(V, E^{\prime}\right)$ containing all the edges of $G$ (i.e., $\left.E \subset E^{\prime}\right)$, on which the maximal peak memory is bounded by $M$.

In general, there exist many possible partial serializations to solve the problem. In particular, one might add so many edges that the resulting graph can only be 
processed sequentially. In order to limit the impact on parallel performance of the partial serialization, we use the critical path length as the metric. The critical path is defined as the path from the source to the sink of the DAG who has the largest total processing time. By minimizing the increase in critical path when adding edges to the graph, we expect that we limit the impact on performance, that is, the increase in makespan when scheduling the modified graph.

We first show that finding a partial serialization of $G$ for memory $M$ is equivalent to finding a sequential schedule executing $G$ using a memory of size at most $M$. On the one hand, given a partial serialization, any topological order is a valid schedule using a memory of size at most $M$. On the other hand, given such a sequential schedule, we can build a partial serialization allowing only this schedule (by adding edge $(i, j)$ if $i$ is executed before $j$ ). Therefore, as finding a sequential schedule executing $G$ using a memory of size at most $M$ is NP-complete by Theorem 4, finding a partial serialization of $G$ for a memory bound of $M$ is also NP-complete.

However, in practical cases, we know that the minimum memory needed to process $G$ is smaller than $M$. Therefore, the need to find such a minimum memory traversal adds an artificial complexity to our problem, as it is usually easy to compute a sequential schedule not exceeding $M$ on actual workflows. We thus propose the following definition of the problem, which includes a valid sequential traversal to the inputs.

Definition 5. The MinPartialSerialization problem, given a DAG $G=(V, E), a$ memory bound $M$, and a sequential schedule $\mathscr{S}$ of $G$ not exceeding the memory bound, consists in computing a partial serialization of $G$ for the memory bound $M$ that has a minimal critical path length.

\subsection{Complexity analysis}

We now show that the MinPartialSerialization problem is NP-complete. As explained above, this complexity does not come from the search of a sequential traversal with minimum peak memory. To prove this result, we first propose the following lower bound on the makespan. 
Lemma 2. Let $G=(V, E)$ be a $D A G$. Any schedule $\mathscr{S}$ of peak memory $M_{\mathscr{S}}$ and makespan $T_{\mathscr{S}}$ verifies:

$$
T_{\mathscr{S}} M_{\mathscr{S}} \geq \sum_{i \in V} \operatorname{Outputs}(i) w_{i} .
$$

As a corollary, if $G$ has a maximal peak memory of $M_{\max }$, then the length $T_{\infty}$ of its critical path satisfies:

$$
T_{\infty} M_{\max } \geq \sum_{i \in V} \text { Outputs }(i) w_{i} .
$$

Proof. To prove this result, we consider the function which associates to each time step the memory usage using schedule $\mathscr{S}$ at this time. Its maximum is $M_{\mathscr{S}}$ and it is defined between $t=0$ and $t=T_{\mathscr{S}}$, so the area under the curve is upper bounded by $T_{\mathscr{S}} M_{\mathscr{S}}$. Now, for each task, its output data must be in memory for at least the execution time of this task; hence, $\sum_{i \in V}$ Outputs $(i) w_{i}$ is a lower bound of the area under the curve, which proves the result.

We now consider the decision version of the MinPartialSERIALIZATIOn problem, which amounts to finding a partial serialization of a graph $G$ for a memory $M$ with critical path smaller than $C P$, and prove that it is NP-complete.

Theorem 8. The decision version of the MinPARTIALSERIALIZATION problem is NPcomplete, even for independent paths of length two.

Proof. This problem is in NP as given a partial serialization of a graph $G$ for a memory bound $M$, one can check in polynomial time that it is valid: simply compute its maximum peak memory (using Algorithm 1) and the length of its critical path.

To prove the problem NP-hard, we perform a reduction from 3-PARTITION, which is known to be NP-complete in the strong sense [32]. We consider the following instance $\mathscr{I}_{1}$ of the 3-PARTITION problem: let $a_{i}$ be $3 m$ integers and $B$ an integer such that $\sum a_{i}=m B$. We consider the variant of the problem, also NP-complete, where $\forall i, B / 4<a_{i}<B / 2$. To solve $\mathscr{I}_{1}$, we need to solve the following question: does there exist a partition of the $a_{i}$ 's in $m$ subsets $A_{1}, \ldots, A_{m}$, each containing exactly 3 elements, such that, for each $A_{k}, \sum_{i \in A_{k}} a_{i}=B$. We build the following instance $\mathscr{I}_{2}$ of our problem. We define a DAG $G$ with $6 m$ vertices denoted by $u_{i}$ and $v_{i}$ for $1 \leq i \leq 3 m$. $G$ contains $3 m$ edges, each pair $\left(u_{i}, v_{i}\right)$, which have weights equal to $a_{i}$. 
Each vertex $u_{i}$ has a unit work and $v_{i}$ has a null work. The memory bound is equal to $B$ and the problem asks whether there exists a partial serialization of $G$ for $B$ with critical path length at most $m$. A schedule $\mathscr{S}$ executing sequentially the pairs $u_{i}, v_{i}$ does not exceed the memory bound $B$ (not even $B / 2$ ), so the instance $(G, B, \mathscr{S})$ is a valid instance of the MinParTiaLSERIALIZATION problem.

Assume first that $\mathscr{I}_{1}$ is solvable, let $A_{1}, \ldots, A_{m}$ be a solution. We build a solution to $\mathscr{I}_{2}$. Define the graph $G^{\prime}$ from the graph $G$ with the following additional edges. For $i \in[1, m-1]$, add edges of null weight between every $v_{j}$ for $a_{j} \in A_{i}$ and every $u_{k}$ for $a_{k} \in A_{i+1}$. The critical path of $G^{\prime}$ is then equal to $m$. Let $S, \bar{S}$ be a topological partition of the graph $G^{\prime}$, with no edge from $\bar{S}$ to $S$, and $C$ be the set of edges between $S$ and $\bar{S}$. Assume that $C$ contains an edge $\left(u_{j}, v_{j}\right): u_{j} \in S$ and $v_{j} \in \bar{S}$. Then let $k$ be such that $a_{j}$ and $a_{k}$ do not belong to the same set $A_{i}$. There is a directed path connecting either $v_{j}$ to $u_{k}$ or $v_{k}$ to $u_{j}$, so $\left(u_{k}, v_{k}\right) \notin C$. Therefore, as $A_{1}, \ldots, A_{m}$ is a solution to $\mathscr{I}_{1}$, the weight of the cut $C$ is equal to $B$, so $G^{\prime}$ solves $\mathscr{I}_{2}$.

Now, assume that $\mathscr{I}_{2}$ is solvable, let $G^{\prime}$ be a partial serialization of $G$ for $B$ whose critical path has length $T_{\infty}$ at most $m$. Note that the following bound due to Lemma 2 is tight on $G^{\prime}$ :

$$
T_{\infty} M_{\max } \geq \sum_{i \in V} \operatorname{Outputs}(i) w_{i}
$$

Indeed, the length of the critical path verifies $T_{\infty} \leq m$, the maximal peak memory $M_{\max }$ verifies $M_{\max } \leq B$, for any $i \in[1, m] u_{i}$ has a unit weight and $v_{i}$ a null one and Outputs $\left(u_{i}\right)=a_{i}$. Therefore, $\sum_{i \in V}$ Outputs $(i) w_{i}=m B$ so $T_{\infty}=m$ and $M_{\max }=B$.

Let $U_{1}$ be the set of nodes $u_{i}$ without predecessors in $G^{\prime}$. There cannot be more than three nodes in $U_{1}$ because the cut $\left(U_{1}, \bar{U}_{1}\right)$ would have a weight larger than $B$. Assume by contradiction that its weight is less than $B$. Consider the graph $G_{1}^{\prime}$ equal to $G^{\prime}$ except that the nodes in $U_{1}$ have a null work. The critical path of $G_{1}^{\prime}$ is equal to $m-1$ and in $G_{1}^{\prime}$, we have $\sum_{i \in V} \operatorname{Outputs}(i) w_{i}>m B-B=(m-1) B$, so the bound of Lemma 2 is violated. Therefore, the weight of the cut $\left(U_{1}, \bar{U}_{1}\right)$ is equal to $B$, so $U_{1}$ is composed of three vertices that we will denote by $u_{i_{1}}, u_{j_{1}}, u_{k_{1}}$, and we have $a_{i_{1}}+a_{j_{1}}+a_{k_{1}}=B$. 
Suppose by contradiction that there exists a node $u_{i}$ not in $U_{1}$ such that there is no path from $v_{i_{1}}, v_{j_{1}}$ or $v_{k_{1}}$ to $u_{i}$. Then, $\left(U_{1} \cup\left\{u_{i}\right\}, V \backslash\left(U_{1} \cup\left\{u_{i}\right\}\right)\right)$ is a topological cut of $G_{1}^{\prime}$ of weight strictly larger than $B$, which is impossible by definition of $\mathscr{I}_{2}$. Therefore, in $G_{1}^{\prime}$, the nodes that have no ancestors are $U_{1}=\left\{u_{i_{1}}, u_{j_{1}}, u_{k_{1}}\right\}$, and the nodes whose ancestors belong in $U_{1}$ are $\left\{v_{i_{1}}, v_{j_{1}}, v_{k_{1}}\right\}$.

We can then apply recursively the same method to determine the second set $U_{2}$ of three vertices $u_{i_{2}}, u_{j_{2}}, u_{k_{2}}$ without ancestors of positive work in $G_{1}^{\prime}$. We now define $G_{2}^{\prime}$ as equal to $G_{1}^{\prime}$ except that nodes of $U_{2}$ have a null work, and continue the induction.

At the end of the process, we have exhibited $m$ disjoint sets of three elements $a_{i}$ that each sum to $B$, so $\mathscr{I}_{1}$ is solvable.

\subsection{Finding an optimal partial serialization through ILP}

We present in this section an Integer Linear Program solving the MinPARTIALSERIALIZATION problem. This formulation combines the linear program determining the maximum topological cut and the one computing the critical path of a given graph.

We consider an instance of the MinPARTIALSERIALIZATION problem, given by a DAG $G=(V, E)$ with weights on the edges, and a memory limit $M$. The sequential schedule $\mathscr{S}$ respecting the memory limit is not required. By convention, for any $(i, j) \notin E$, we set $m_{i, j}=0$.

We first consider the $e_{i, j}$ variables, which are equal to 1 if edge $(i, j)$ exists in the associated partial serialization, and to 0 otherwise.

$$
\begin{gathered}
\forall(i, j) \in V^{2}, \quad e_{i, j} \in\{0,1\} \\
\forall(i, j) \in E, \quad e_{i, j}=1 .
\end{gathered}
$$

We need to ensure that no cycle has been created by the addition of edges. For this, we compute the transitive closure of the graph: we enforce that the graph contains edge $(i, j)$ if there is a path from node $i$ to node $j$. Then, we know that the graph is acyclic if and only if it does not contain any self-loop. This corresponds to 
the following constraints:

$$
\begin{gathered}
\forall(i, j, k) \in V^{3}, \quad e_{i, k} \geq e_{i, j}+e_{j, k}-1 \\
\forall i \in V, \quad e_{i, i}=0 .
\end{gathered}
$$

Then, we use the flow variables $f_{i, j}$, in a way similar to the formulation of the MinFLow problem. If $e_{i, j}=1$, then $f_{i, j} \geq m_{i, j}$, and $f_{i, j}$ is null otherwise. Now, the flow going out of $s$ is equal to the maximal cut of the partial serialization, see the proof of Theorem 6, so we ensure that it is not larger than $M$. Now, note that each $f_{i, j}$ can be upper bounded by $M$ without changing the solution space. Therefore, Equation (11) ensures that $f_{i, j}$ is null if $e_{i, j}$ is null, without adding constraints on the others $f_{i, j}$. This leads to the following inequalities:

$$
\begin{gathered}
\forall(i, j) \in V^{2}, \quad f_{i, j} \geq e_{i, j} m_{i, j} \\
\forall(i, j) \in V^{2}, \quad f_{i, j} \leq e_{i, j} M \\
\forall j \in V \backslash\{s, t\}, \quad \sum_{i \in V} f_{i, j}-\sum_{k \in V} f_{j, k}=0 \\
\sum_{j \in V} f_{s, j} \leq M .
\end{gathered}
$$

This set of constraints defines the set of partial serializations of $G$ with a maximal cut at most $M$. It remains to compute the length of the critical path of the modified graph, in order to formalize the objective. We use the variables $p_{i}$ to represent the top-level of each task, that is, their earliest completion time in a parallel schedule with infinitely many processors. The completion time of task $s$ is $w_{s}$, and the completion time of another task is equal to its processing time plus the maximal completion time of its predecessors:

$$
\begin{aligned}
p_{s} & \geq w_{s} \\
\forall(i, j) \in V^{2}, \quad & p_{j} \geq w_{j}+p_{i} e_{i, j} .
\end{aligned}
$$

The previous equation is not linear, so we transform it by using $W$, the sum of the processing times of all the tasks and the following constraints.

$$
\begin{aligned}
\forall i \in V, & p_{i} \geq w_{i} \\
\forall(i, j) \in V^{2}, & p_{j} \geq w_{j}+p_{i}-W\left(1-e_{i, j}\right) .
\end{aligned}
$$


If $e_{i, j}$ is null, then Equation (15) is less restrictive than Equation (14) as $p_{i}<W$, which is expected as there is no edge $(i, j)$ in the graph. Otherwise, we have $e_{i, j}=1$ and the constraints on $p_{j}$ are the same as above.

Finally, we define the objective as minimizing the top-level of $t$, which is the critical path of the graph.

$$
\text { Minimize } p_{t} \quad \text { under Equations (6) to (15). }
$$

We denote $\mathscr{P}$ the resulting ILP. We now prove that there exists a solution to $\mathscr{P}$ of objective at most $L$ if and only if there exists a partial serialization $P S$ of $G$ with memory bound $M$ of critical path length at most $L$.

Consider a solution of $\mathscr{P}$ of objective cost at most $L$. Let $P S$ be the directed graph composed of the edges $(i, j)$ for every $i, j \in V^{2}$ such that $e_{i, j}=1$. The weight of such edges is $m_{i, j}$. We can show by induction on the size of a potential cycle that $P S$ is acyclic. No self-loop can exist as all $e_{i, i}$ are null. If a cycle contains more than one edge, Equation (8) ensures the existence of a strictly smaller cycle, while Equation (9) forbids self-loops. Then, the equations concerning $f_{i, j}$ model the MinFLow problem already studied, and ensure that the minimum flow is smaller than $M$. The only difference being that each $f_{i, j}$ is bounded by $M$, which is already the case in any solution. Finally, consider a critical path $\left(s, i_{1}, i_{2}, \ldots, i_{k}, t\right)$ of $P S$. The equations concerning the variables $p_{i}$ ensure that $p_{t} \geq w_{s}+w_{i_{1}}+\cdots+w_{i_{k}}+w_{t}$. Therefore, $L$ is not smaller than the critical path length. Therefore, $P S$ is a partial serialization for $M$ of critical path length at most $L$.

Now, consider a partial serialization $P S$ of $G$ for $M$, of critical path length at most $L$. We set $e_{i, j}=1$ if and only if there exists a path from $i$ to $j$ in $P S$. This respects the acyclicity constraints as $P S$ is a DAG by definition. The maximum peak memory of $P S$ is at most $M$, therefore the maximum cut of the graph induced by the variables $e_{i, j}$ is at most $M$, so there exists a valuation of the variables $f_{i, j}$ satisfying the flow constraints. Finally, we set the variables $p_{i}$ equal to the top-level of task $i$ in PS:

$$
\forall i \in V, \quad p_{i}=w_{i}+\max _{j \in V}\left\{e_{j, i} p_{j}\right\}
$$

This valuation satisfies the last constraints and the objective function is then equal 
to $L$.

\subsection{Heuristic strategies to compute a partial serialization}

We now propose several heuristics to solve the MINPARTIALSERIALIZATION problem. These heuristics are based on a same framework, detailed in Algorithm 2. The idea of the algorithm, inspired by [8], is to iteratively build a partial serialization $G^{\prime}$ from $G$. At each iteration, the topological cut of maximum weight is computed via Algorithm 1. If its weight is at most $M$, then the algorithm terminates, as the obtained partial serialization is valid. Otherwise, another edge has to be added in order to reduce the maximum peak memory. We rely on a subroutine in order to choose which edge to add. In the following, we propose four possible subroutines. If the subroutine succeeds to find an edge that does not create a cycle in the graph, we add the chosen edge to the current graph. Otherwise, the heuristic fails. Such a failure may happen if the previous choices of edges have led to a graph which is impossible to schedule without exceeding the memory.

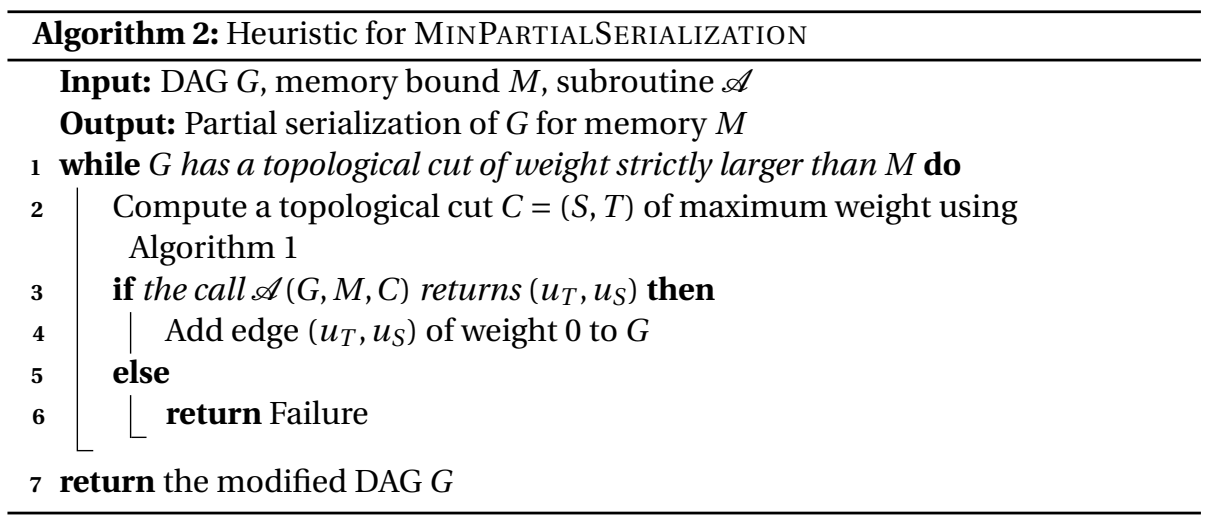

We propose four possibilities for the subroutine $\mathscr{A}(G, M, C)$, which selects an edge to be added to $G$. They all follow the same structure: two vertices $u_{S}$ and $u_{T}$ are selected from the maximum cut $C=(S, T)$, where $u_{S} \in S$ and $u_{T} \in T$ and no path exists from $u_{S}$ to $u_{T}$. The returned edge is then $\left(u_{T}, u_{S}\right)$. For instance, in the toy example of Figure 1, only two such edges can be added: $(c, b)$ and $(d, a)$. Note that adding such an edge prevents $C$ from remaining a valid topological cut, thus it is 
likely that the weight of the new maximum topological cut will be reduced. Note also that adding such an edge cannot create a cycle.

We first recall some classical attributes of a graph:

- The length of a path is the sum of the work of all the nodes in the path, including its extremities;

- The bottom-level of an edge $(i, j)$ or a node $i$ is the length of the longest path from $i$ to $t$ (the sink of the graph);

- The top-level of an edge $(i, j)$ or of a node $j$ is the length of the longest path from $s$ (the source of the graph) to $j$, excluding the work of $j$.

We now present the four subroutines. The MinLEVELS heuristic, as well as the two following ones, considers the set $P$ of vertex couples $(j, i) \in T \times S$ such that no path from $i$ to $j$ exist. Note that $P$ corresponds to the set of candidate edges that might be added to $G$. Then, it returns the couple $\left(u_{T}, u_{S}\right) \in P$ that optimizes a given metric. If $P$ is empty, then the subroutine fails. MinLevels tries to minimize the critical path of the graph obtained when adding the new edge, by preventing the creation of a long path from $s$ to $t$. Thus, it returns the couple $(j, i) \in P$ that minimizes top_level $(j)+$ bottom_level $(i)$.

The MAXSIzE heuristic aims at minimizing the weight of the next topological cut. Thus, it selects a couple $(j, i)$ such that outgoing edges of $i$ and incoming edges of $j$ contribute a lot to the weight of the current cut. Formally, it returns the couple $(j, i) \in P$ that maximizes $\sum_{k \in T} m_{i, k}+\sum_{k^{\prime} \in S} m_{k^{\prime}, j}$ (considering that $m_{i, j}=0$ if there is no edge from $i$ to $j$ ).

The MAXMinSize heuristic is a variant of the previous heuristic and pursues the same objective. However, it selects a couple of vertices which both contribute a lot to the weight of the cut, by returning the couple $(j, i) \in P$ that maximizes $\min \left(\sum_{k \in T} m_{i, k}, \sum_{k^{\prime} \in S} m_{k^{\prime}, j}\right)$.

Finally, the last heuristic is the only one that is guaranteed to never fail. To achieve this, it relies on a sequential schedule $\mathscr{S}$ of the graph that does not exceed the memory $M . \mathscr{S}$ is defined by a function $\sigma$, where $\sigma(i)$ equals the starting time of task $i$ in $\mathscr{S}$. Such a sequential schedule needs to be precomputed, and we propose a possible algorithm below. 
Given such a sequential schedule $\mathscr{S}$, this heuristic, named RespeCtORDER, always adds an edge ( $j, i)$ which is compatible with $\mathscr{S}$ (i.e., such that $\sigma(j) \leq \sigma(i)$ ), and which is likely to have the smallest impact on the set of valid schedules for the new graph, by maximizing the distance $\sigma(i)-\sigma(j)$ from $j$ to $i$ in $\mathscr{S}$. Let $u_{T}$ be the node of $T$ which is the first to be executed in $\mathscr{S}$, and $u_{S}$ be the node of $S$ which is the last to be executed in $\mathscr{S}$. Note that $u_{S}$ must be executed after $u_{T}$ in $\mathscr{S}$, because otherwise, the peak memory of $\mathscr{S}$ will be at least the weight of $C$ which is a contradiction. The returned couple is then $\left(u_{T}, u_{S}\right)$. Note that no path from $u_{S}$ to $u_{T}$ can exist in the graph if all the new edges have been added by this method. Indeed, all the added edges respect the order $\mathscr{S}$ by definition. Then, no failure is possible, but the quality of the solution highly depends on the input schedule $\mathscr{S}$.

\subsection{Computing a sequential schedule for MINLEVELS}

In this section we discuss the generation of the schedule $\mathscr{S}$, which is used as an input for heuristic RESPECTORDER. By definition, this sequential schedule executes the DAG $G$ using a memory at most $M$. As proven in Theorem 4 , deciding if such a schedule exists is NP-complete. However, most graphs describing actual workflows exhibit a high level of parallelism, and the difficulty is not in finding a sequential schedule fitting in memory. As a consequence, we assume that a Depth First Search (DFS) schedule, which always completes a parallel branch before starting a new one, never exceeds the memory bound.

The problem with a DFS schedule is that applying RESPECTORDER using such a schedule is likely to produce a graph with a large critical path. For this objective, a Breadth First Search (BFS) schedule is more appropriate, but it is not likely to respect the memory bound.

As proposed in [8], a way to solve this problem is to "mix" DFS and BFS schedules, and tune the proportion of each one to get a schedule respecting the memory bound but still offering good opportunities for parallelism. Formally, we define the $\alpha$-BFsDFs schedule, which depends on the parameter $\alpha \in[0,1]$ and two schedules, a DFS and a BFS. A 0-BFSDFS schedule is equal to the BFS and a 1-BFSDFs schedule is equal to the DFS. For a given task $i$, we note $D F S(i)$ and $B F S(i)$ the rank of task $i$ 
according to each schedule (i.e., the number of tasks executed before task $i$ ). Then, the $\alpha$-BFSDFS schedules the tasks of $G$ in non-decreasing order of

$$
\alpha D F S(i)+(1-\alpha) B F S(i)
$$

The $\alpha$-BFSDFS schedule respects the precedence constraints: indeed, if task $i$ has a successor $j$, then $i$ is scheduled before $j$ in both BFS and DFS. Then, as $\alpha$ and $1-\alpha$ are non-negative, $\alpha$-BFSDFS schedules $i$ before $j$.

The idea consists in starting from the 0-BFsDFs schedule, and then to increase the $\alpha$ parameter until the memory of the resulting schedule is not larger than $M$. As we assumed that DFS (1-BFSDFS) does not exceed $M$, this process is guaranteed to success. In practice, we chose in the experiments to increment $\alpha$ by step of 0.05 until we find an appropriate schedule.

\section{Simulation results}

We now compare the performance of the proposed heuristics through simulations on synthetic DAGs. All heuristics are implemented in C++ using the igraph library [33].

\begin{tabular}{crrrrr}
\hline & \multicolumn{2}{c}{ DagGen } & Ligo & Montage & Genome \\
& dense & sparse & & & \\
\hline Nb. of test cases & 572 & 572 & 220 & 220 & 220 \\
\hline MinLEVELS & 1 & 12 & 20 & 1 & 0 \\
RESPECTORDER & 0 & 0 & 0 & 0 & 0 \\
MAXMinSizE & 2 & 5 & 3 & 0 & 0 \\
MAXSizE & 6 & 12 & 13 & 0 & 17 \\
ILP & 26 & 102 & & & \\
\hline
\end{tabular}

Table 1: Number of failures for each dataset.

We generated the first dataset, named DAGGEN, using the DAGGEN software [34]. Five parameters influence the generation of these DAGs. The number of nodes belongs to $\{25,50,100\}$. The width, which controls how many tasks may run in parallel, belongs to $\{0.2,0.5,0.8\}$. The regularity, which controls the distribution of the tasks between the levels, belongs to $\{0.2,0.8\}$. The density, which controls how 
many edges connect two consecutive levels, belongs to $\{0.2,0.8\}$. The jump, which controls how many levels an edge may span, belongs to $\{1,2,4\}$. Combining all these parameters, we obtain a dataset of 108 DAGs. This dataset has already been used to model workflows in the scheduling literature [35,36]. We split it in two parts in the representations: the sparse DAGGEN dataset contains the DAGs with a density of 0.2 and the dense DAGGEN dataset contains the DAGs with a density of 0.8 . Indeed, this parameter leads to significant differences in the results; hence, the distinction.

The three other datasets represent actual applications and have been generated with the Pegasus Workflow Generator [37]. We consider three different datasets, named Ligo, Montage, and Genome, each containing 20 graphs of 100 nodes. We assumed that the memory needed during the execution of a node is negligible compared to the size of the input and output data, which must be kept in memory during this process. As some produced data may be shared between several tasks, we apply the transformation presented in Section 3.

The heuristics have been simulated for eleven memory bounds per DAG, evenly spread between two bounds. Only half of them are represented in the plots for space reasons. The smallest bound corresponds to the memory required for a DFS schedule, while the largest bound corresponds to the maximal peak memory of the DAG. In the results, a normalized memory of 0 corresponds to the smallest bound, while 1 corresponds to the largest bound.

One may argue that the range of memory considered can be small for some graphs, and will then be of little interest. We therefore computed the ratio of the largest memory considered divided by the smallest for each graph, and we present the statistic summary in Table 2. We can see that this ratio is very high for the LIGO and GENOME dataset: finding a partial serialization achieving the smallest memory bound means that the maximal memory consumption is divided by more than 20 for most of these graphs. This ratio has a median of 6 for the MONTAGE, which is also a high potential improvement. It is lower for the sparse DAGGEN dataset, with a median of 2, and especially for the sparse DAGGEN dataset, with a median of 1.3. Note that 4 DAGs of the DAGGEN dataset have been discarded because the minimum memory equals the maximum memory. 


\begin{tabular}{cccccc}
\hline & \multicolumn{2}{c}{ DagGen } & Ligo & Montage & GEnome \\
& dense & sparse & & & \\
\hline First quartile & 1.2 & 1.7 & 21.2 & 5.5 & 20.1 \\
Median & 1.3 & 2 & 21.7 & 6.2 & 21.5 \\
Third quartile & 1.4 & 2.5 & 22.1 & 6.8 & 22 \\
\hline
\end{tabular}

Table 2: Statistic summary of the ratio maxmem/minmem for each dataset.

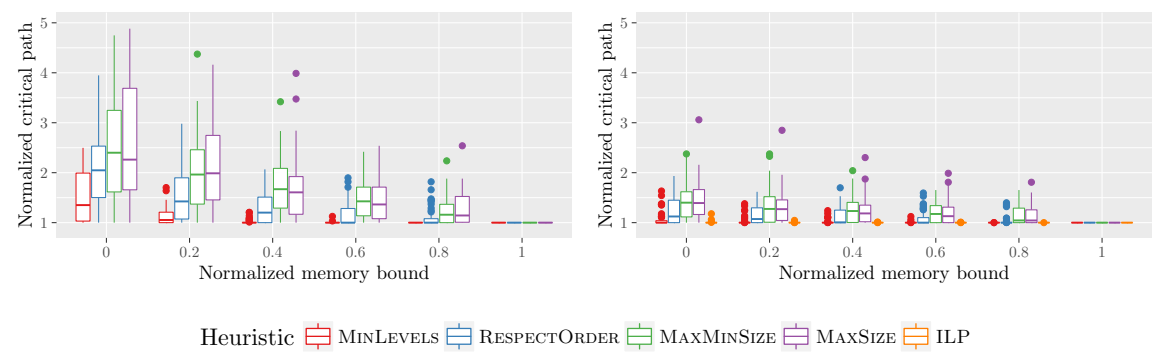

Figure 4: Critical path length obtained by each method for the DAGGEN dataset (left:sparse, right:dense).

Critical path is normalized by the one of the original graph. Memory normalized so that 0 is DFS

schedule and 1 is original maximum peak memory.

In order to assess the performance of the heuristics, we first examine the critical path length of the obtained partial serialization. We normalize each critical path by the critical path of the original graph. Therefore, for the largest memory bounds, the original graph being itself a valid partial serialization, all the normalized critical paths equal 1 . When a method fails to find a solution, we say that the critical path achieved is infinite. As we focus on the statistical summary of the results (rather than on the average), this allows to fairly compare two heuristics with different success rate, as only the outlier points are not displayed. Failure rates are reported in Table 1.

We plot the results obtained for the sparse and dense DAGGEN dataset in Figure 4. For each heuristic and memory bound, we display the 108 results as a Tukey boxplot. The box presents the median, the first and third quartiles. The whiskers extend to up to 1.5 times the box height, and points outside are plotted individually. The first trend that can be observed, is that, as expected, the lower the memory bound, the larger the critical path. The difference between the minimal and the maximal memory bound is smaller for dense graphs. Therefore, it is logical that the 
heuristics lead to a larger increase of the critical path in sparse graphs. Comparing the heuristics, we can see that MINLEVELS clearly outperforms the other ones for any value of the memory bound. Then, RESPECTORDER obtains better performance than MAXMINSIZE and MAXSIZE. Note that no significant difference appears when restricting the dataset to specific values of the generation parameters. The results are widely spread as the graphs differ in several parameters. We remark therefore that MinLevels is highly robust considering the variety of the graphs. On this dataset, we have also computed the optimal solution by using the Integer Linear Program presented in Section 5. We implemented the ILP using CPLEX with a time limit of one hour of computation on a standard laptop computer (8 cores Intel i7). When it was unable to provide a solution within the time limit, we assume a failure. This happens on sparse graphs, especially for low memory bounds, which is why it is omitted on Figure 4, left. The exact failure rates are reported in Table 1.

We also studied the makespan obtained by a classic scheduler for a given number of processors on the graphs returned by each heuristic. The results are expectedly similar to the ones obtained by focusing on the critical path and have then been deferred to the companion research report [21].
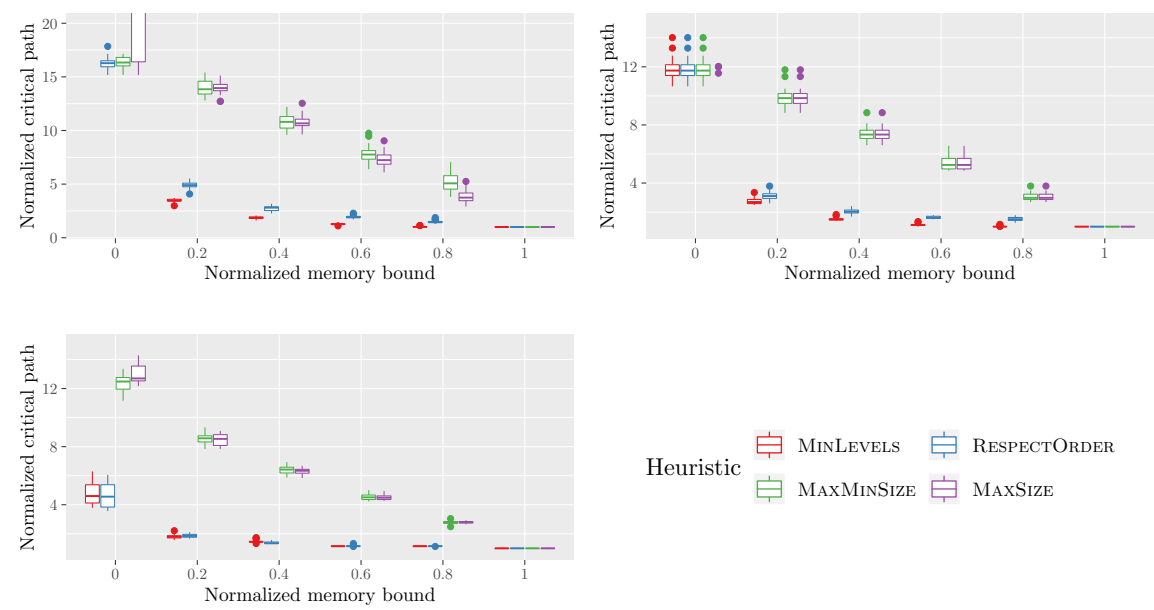

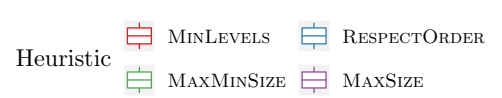

Figure 5: Critical path length obtained by each method for the LIGO (top left), GENOME (top right) and MONTAGE (bottom) datasets. Critical path and memory normalized as in Figure 4. 
We plot the results obtained for the LIGO, GENOME and MONTAGE dataset on Figure 5, showing the critical path lengths achieved by each heuristic for each memory bound. The similar structure of all graphs for each of these datasets explains that the results lie in a smaller interval. The hierarchy of the heuristics is the same as in the DAGGEN dataset: MinLEVELS presents the best performance, RESPECTORDER leads to slightly longer critical paths, and MAXSIZE and MAXMINSIZE achieve similar results, several times higher than the first two heuristics. Note that for the smallest memory bound, MinLEVELS never succeeds in the LIGO dataset (hence, it does not appear in the plot), MAXSIZE also presents a high failure rate, whereas RESPECTORDER and MAXMINSIZE have comparable results. We observe a similar trend for the GENOME dataset, except that MinLEVELS never fails, even for the smallest memory bound. For the MONTAGE dataset, the trend is again similar, except that MINLEVELS and RESPECTORDER always present better results than the other heuristics, even for the smallest memory bound.

We have shown in these experiments that we can partially serialize realistic graphs so that any schedule fits a given memory bound, for a reasonable cost in terms of the critical path and makespan augmentation. One may argue that the maximal peak memory considered does not reflect the actual memory consumption of a traditional algorithm. In order to address this problem, we measured the peak memory achieved by the Earliest Finish Time (EFT) scheduling heuristic which, whenever a task terminates, schedules the available task with the highest bottom level. Then, we normalized it in the same way as in the plots above: a value of 1 means that the maximal peak memory is actually achieved, and a value of 0 means that the peak memory reached is the same as the Depth First Search considered. Note that we can obtain negative values, which happened only for some graphs of the DAGGEN datasets, if the DFS requires a larger memory than EFT. The statistical summary is presented in Table 3. We note that EFT uses the maximal peak memory for most of the graphs of the LIGO and GENOME datasets, and a normalized memory larger than 0.88 for most of the graphs of the MONTAGE dataset. We recall that from Table 2, the ratio between maximum memory and the DFS memory is around 21.5 for Ligo and Genome and 6.2 for Montage. Therefore, on these graphs, low- 
ering the maximum memory peak can lead to a dramatic reduction of the memory consumption when using scheduling heuristics such as EFT.

On the DAGGEN dataset, the partial serialization is not as beneficial, as we obtain a median of 0.53 for the normalized memory of EFT. Besides, the ratio between DFS and BFS memory is also smaller. Thus, the gain in memory of using partial serialization is less impressive, although noticeable.

\begin{tabular}{crllll}
\hline & \multicolumn{2}{c}{ Daggen } & Ligo & Montage & Genome \\
& dense & sparse & & & \\
\hline First quartile & -0.03 & 0.39 & 0.99 & 0.88 & 1 \\
Median & 0.31 & 0.6 & 1 & 0.9 & 1 \\
Third quartile & 0.71 & 0.75 & 1 & 0.93 & 1 \\
\hline
\end{tabular}

Table 3: Normalized memory used by EFT. Memory normalized as in Figure 4.

\section{Conclusion}

In this paper, we have focused on lowering the memory footprint of computational workflows modeled as task graphs. As we recognized the need for dynamic schedules (such as in runtime systems), we have focused on the transformation of the graphs prior to the scheduling phase. Adding fictitious edges that represent "memory dependences" prevents the scheduler from running out of memory. After formally modeling the problem, we have shown how to compute the maximal peak memory of a graph (the MAXTOPCUT problem) in polynomial time. We have proven the problem of adding edges to cope with limited memory while minimizing the critical path (the MinPartialSerialization problem) to be NP-hard, and proposed both an ILP formulation of the problem and several heuristics. Our simulations show that our best heuristics, RESPECTORDER and MinLEVELS, either never fail, or are able to limit the memory footprint with limited impact on the parallel makespan for most task graphs. Our future work consists in implementing the proposed heuristics in a runtime system and evaluate them on actual graphs. 
[1] M. Drozdowski, Scheduling parallel tasks - algorithms and complexity, in: J. Leung (Ed.), Handbook of Scheduling, Chapman and Hall/CRC, 2004.

[2] C. Augonnet, S. Thibault, R. Namyst, P.-A. Wacrenier, StarPU: a unified platform for task scheduling on heterogeneous multicore architectures, Concurrency and Computation: Practice and Experience 23 (2) (2011) 187-198.

[3] T. Gautier, X. Besseron, L. Pigeon, KAAPI: A thread scheduling runtime system for data flow computations on cluster of multi-processors, in: International Workshop on Parallel Symbolic Computation, 2007, pp. 15-23.

[4] J. Planas, R. M. Badia, E. Ayguadé, J. Labarta, Hierarchical task-based programming with StarSs, IJHPCA 23 (3) (2009) 284-299.

[5] G. Bosilca, A. Bouteiller, A. Danalis, M. Faverge, T. Herault, J. J. Dongarra, PaRSEC: Exploiting heterogeneity for enhancing scalability, Computing in Science \& Engineering 15 (6) (2013) 36-45.

[6] A. Ramakrishnan, G. Singh, H. Zhao, E. Deelman, R. Sakellariou, K. Vahi, K. Blackburn, D. Meyers, M. Samidi, Scheduling data-intensiveworkflows onto storage-constrained distributed resources, in: CCGrid’07, 2007, pp. 401-409.

[7] E. Agullo, P. R. Amestoy, A. Buttari, A. Guermouche, J. L'Excellent, F. Rouet, Robust memory-aware mappings for parallel multifrontal factorizations, SIAM J. Scientific Computing 38 (3).

[8] D. Sbîrlea, Z. Budimlić, V. Sarkar, Bounded memory scheduling of dynamic task graphs, in: Proc. of PACT, ACM, 2014, pp. 343-356.

[9] L. Marchal, H. Nagy, B. Simon, F. Vivien, Parallel scheduling of dags under memory constraints, in: 2018 IEEE International Parallel and Distributed Processing Symposium (IPDPS), 2018, pp. 204-213.

[10] R. Sethi, J. Ullman, The generation of optimal code for arithmetic expressions, Journal of the ACM 17 (4) (1970) 715-728. 
[11] R. Sethi, Complete register allocation problems, in: STOC'73, ACM Press, 1973, pp. 182-195.

[12] J. R. Gilbert, T. Lengauer, R. E. Tarjan, The pebbling problem is complete in polynomial space, SIAM J. Comput. 9 (3).

[13] J. W. H. Liu, An application of generalized tree pebbling to sparse matrix factorization, SIAM J. Alg. Discrete Methods 8 (3) (1987) 375-395.

[14] C.-C. Lam, T. Rauber, G. Baumgartner, D. Cociorva, P. Sadayappan, Memoryoptimal evaluation of expression trees involving large objects, Computer Languages, Systems \& Structures 37 (2) (2011) 63-75.

[15] L. Eyraud-Dubois, L. Marchal, O. Sinnen, F. Vivien, Parallel scheduling of task trees with limited memory, ACM Transactions on Parallel Computing 2 (2) (2015) 13.

[16] S. Bharathi, A. Chervenak, Scheduling data-intensive workflows on storage constrained resources, in: Proc. of the 4th Workshop on Workflows in Support of Large-Scale Science (WORKS'09), ACM, 2009.

[17] M. Sergent, D. Goudin, S. Thibault, O. Aumage, Controlling the memory subscription of distributed applications with a task-based runtime system, in: Proc. of IPDPS Workshops, IEEE, 2016, pp. 318-327.

[18] Z. Budimlić, M. Burke, V. Cavé, K. Knobe, G. Lowney, R. Newton, J. Palsberg, D. Peixotto, V. Sarkar, F. Schlimbach, et al., Concurrent collections, Scientific Programming 18 (3-4) (2010) 203-217.

[19] S. Touati, Register Pressure in Instruction Level Parallelism, Theses, Université de Versailles-Saint Quentin en Yvelines (Jun. 2002).

[20] M. Jacquelin, L. Marchal, Y. Robert, B. Uçar, On optimal tree traversals for sparse matrix factorization, in: Proc. of the Int. Par. \& Dist. Processing Symposium (IPDPS), IEEE, 2011, pp. 556-567. 
[21] L. Marchal, B. Simon, F. Vivien, Limiting the memory footprint when dynamically scheduling DAGs on shared-memory platforms, Research Report RR9231, INRIA, https://hal .inria.fr/hal-01948462 (Dec. 2018).

[22] R. Sethi, Complete register allocation problems, SIAM journal on Computing 4 (3) (1975) 226-248.

[23] E. L. Lawler, Combinatorial optimization: networks and matroids, Courier Corporation, 2001.

[24] R. M. Karp, Reducibility among combinatorial problems, in: Complexity of computer computations, Springer, 1972, pp. 85-103.

[25] M. Garey, D. Johnson, L. Stockmeyer, Some simplified np-complete graph problems, Theoretical Computer Science 1 (3) (1976) 237 - 267.

[26] M. Lampis, G. Kaouri, V. Mitsou, On the algorithmic effectiveness of digraph decompositions and complexity measures, Discrete Optimization 8 (1) (2011) $129-138$.

[27] Peter Shor (http://cs.stackexchange.com/users/198/peter-shor), Minimum s-t cut in weighted directed acyclic graphs with possibly negative weights, Computer Science Stack Exchange. arXiv:http://cs.stackexchange.com/q/6498.

URL http://cs. stackexchange.com/q/6498

[28] T. H. Cormen, C. E. Leiserson, R. L. Rivest, C. Stein, Introduction to Algorithms, Third Edition, 3rd Edition, The MIT Press, 2009.

[29] E. Ciurea, L. Ciupalâ, Sequential and parallel algorithms for minimum flows, Journal of Applied Mathematics and Computing 15 (1) (2004) 53-75.

[30] A. V. Goldberg, R. E. Tarjan, A new approach to the maximum flow problem, in: Proceedings of ACM STOC, 1986, pp. 136-146.

[31] S.-A.-A. Touati, Register saturation in instruction level parallelism, International Journal of Parallel Programming 33 (4) (2005) 393-449. 
[32] M. R. Garey, D. S. Johnson, Computers and Intractability, a Guide to the Theory of NP-Completeness, W. H. Freeman and Company, 1979.

[33] G. Csardi, T. Nepusz, The igraph software package for complex network research, InterJournal Complex Systems (2006) 1695.

URL http: //igraph.org

[34] F. Suter, Daggen: A synthetic task graph generator, https://github.com/ frs69wq/daggen.

[35] S. Hunold, One step toward bridging the gap between theory and practice in moldable task scheduling with precedence constraints, Concurrency and Computation: Practice and Experience 27 (4) (2015) 1010-1026.

[36] F. Desprez, F. Suter, A bi-criteria algorithm for scheduling parallel task graphs on clusters, in: CCGrid, IEEE, 2010, pp. 243-252.

[37] R. F. Da Silva, W. Chen, G. Juve, K. Vahi, E. Deelman, Community resources for enabling research in distributed scientific workflows, in: 10th Int. Conf. on eScience, Vol. 1, IEEE, 2014, pp. 177-184. 